\title{
Review: cognitive behavioural interventions improve some sleep outcomes in older adults
}

Montgomery P,Dennis J. Cognitive behavioural interventions for sleep problems in adults aged 60+. Cochrane Database Syst Rev 2002;(2):CD003161 (latest version 13 Dec 2001).

QUESTION: In adults who are $\geq 60$ years of age, are cognitive behavioural interventions (CBIs) effective for treating sleep problems?

\section{Data sources}

Studies were identified by searching Medline, CINAHL EMBASE/Excerpta Medica, PsycINFO, the Cochrane Controlled Trials Register, the National Research Register, and a sleep bibliography (www.websciences.org/ bibliosleep); handsearching reference lists; and contacting experts in the field.

\section{Study selection}

2 reviewers independently selected randomised controlled trials (RCTs) that compared any form of CBI with a waiting list control or placebo in adults who were $\geq 60$ years of age and had been diagnosed with sleep problems according to standardised or objective measures or sleep diaries. Studies of patients with depression, dementia, sleep apnoea, or sleep problems secondary to another disorder were excluded.

\section{Data extraction}

2 reviewers independently extracted data on patients, interventions, and outcomes (including sleep onset latency time, wake time after sleep onset, sleep duration, sleep efficiency, and quality of sleep).

\section{Main results}

6 RCTs (282 patients, mean age 66 y) met the selection criteria. CBI reduced the amount of wake time after sleep onset and total wake time immediately after treatment (table); the treatment effect did not exist at 3 or $\geq 12$ months. Sleep duration was longer for CBI than for control at $\geq 12$ months when assessed by diaries (table); the treatment effect was not present after treatment or at 3 months or when measured by polysomnography. The sleep efficiency ratio measured by polysomnography was higher in the CBI group than the control group after treatment; the difference was not statistically significant at $\geq 12$ months or when measured by diaries. Patients in the CBI group had better scores on the Pittsburgh Sleep Quality Index than the control group after treatment and at 3 months (table). The groups did not differ for sleep onset latency or early morning awakening.

\section{Conclusion}

In adults who are $\geq 60$ years of age, cognitive behavioural interventions lead to short term improvements in sleep quality, sleep efficiency, and wake time, and to long term improvement in self reported sleep duration.

\section{COMMENTARY}

Sleep problems become more common with age. Because insomnia and the consequent sleep deficit may compromise wellbeing and worsen age related chronic disorders, ${ }^{1}$ it is important to routinely screen for sleep problems in clinical practice. Psychological interventions using cognitive and behavioural strategies include a range of treatments that are usually easy to apply and inexpensive.

Previous meta-analyses support the use of behavioural interventions for improving sleep in younger people. For primary long term insomnia, treatment outcomes have been positive, but short lived, with efficacy similar to benzodiazepines or their receptor agonists. ${ }^{2-4}$ However, it is unclear whether such non-pharmacological interventions are effective for older people. The systematic review by Montgomery and Dennis shows that CBIs slightly improve sleep problems (particularly sleep maintenance insomnia) in elderly people.

Based on the findings of this review, cognitive behavioural therapies appear to be a tempting option for the treatment of insomnia, regardless of a patient's age. However, readers should note that primary insomnia with no comorbid conditions, such as substance use or personality disorders, is relatively rare. The exclusion of people with depressive disorder, dementia, sleep apnoea, or secondary insomnia does not reflect the diversity of everyday life. A need still exists for a review on the effectiveness of CBIs for people with insomnia inclusive of all relevant comorbid conditions.

Timo Partonen, $\mathrm{MD}, \mathrm{PhD}$

National Public Health Institute, Helsinki, Finland

1 Spiegel K, Leproult R, Van Cauter E. Impact of sleep debt on metabolic and endocrine function. Lancet 1999;354:1435-9. Morin CM, Culbert JP, Schwartz SM. Nonpharmacological interventions for insomnia: a meta-analysis of treatment efficacy. Am J Psychiatry 1994:151:1172-80.

3 Murtagh DR, Greenwood KM. Identifying effective psychological treatments for insomnia: a meta-analysis. $J$ Consult Clin Psychol 1995;63:79-89.

4 Smith MT, Perlis ML, Park A, et al. Comparative meta-analysis of pharmacotherapy and behavior therapy for persistent insomnia. Am J Psychiatry 2002;159:5-11.

paul.montgomery@

psych.ox.ac.uk

Cognitive behavioural interventions (CBIs) v wait list or placebo for sleep problems in older adults

\begin{tabular}{|c|c|c|c|c|}
\hline \multirow[b]{2}{*}{ Outcomes } & \multirow[b]{2}{*}{$\begin{array}{l}\text { Number of } \\
\text { trials }\end{array}$} & \multicolumn{2}{|c|}{ Weighted mean scores } & \multirow[b]{2}{*}{$\begin{array}{l}\text { Weighted mean } \\
\text { difference }(95 \% \mathrm{CI})^{\star}\end{array}$} \\
\hline & & CBI & Control & \\
\hline Wake after sleep onset at treatment end (diaries) ( $\mathrm{min}$ ) & 4 & 35.8 & 57.7 & $21.9(6.4$ to 37.3$)$ \\
\hline Wake after sleep onset at treatment end (polysomnography) (min) & 2 & 4.8 & 59.1 & $24.4(7.6$ to 41.1$)$ \\
\hline Total wake time at treatment end (diaries) (min) & 1 & $70.5 \dagger$ & $132.8 \dagger$ & $62.2(16.5$ to 107.9$) \dagger$ \\
\hline Total wake time at treatment end (polysomnography) (min) & 1 & $63.1 \dagger$ & $101.0 \dagger$ & $37.9(7.8$ to 68.1$) \dagger$ \\
\hline Sleep duration at $\geq 12$ months (diaries) (min) & 2 & 400.6 & 361.5 & 39.1 (7.4 to 70.7$)$ \\
\hline Pittsburgh Sleep Quality Index at treatment end & 1 & $7.8 \dagger$ & $10.6 \dagger$ & $2.8(0.2$ to 5.4$) \dagger$ \\
\hline Pittsburgh Sleep Quality Index at 3 months & 1 & $6.2 \dagger$ & $10.2 \dagger$ & $4.0(1.4$ to 6.6$) \dagger$ \\
\hline
\end{tabular}

${ }^{*}$ All mean differences favour $\mathrm{CBI}$.

tData not weighted. 